\title{
Variation of Variability in TEC for Quiet and Disturbed Days at Low Latitude Station Bhopal using GPS
}

\author{
Dr. Kalpana Maski ${ }^{1}$ and Prof. S.K. Vijay ${ }^{2}$
}

\begin{abstract}
GPS is a commanding tool to probe ionospheric variability. Regardless of our improved knowledge of the ionosphere dynamics, the day-to-day variability still lies within the framework of statistical estimations and the underlying physical mechanisms are far from being fully understood. Significant deviations from monthly median values are observed from time to time in ionospheric records during completely quiet solar and geophysical conditions and are not fully understood.

TEC obtained from GPS signals are used to study ionospheric dynamics over Bhopal during low solar activity period March 2004 -February 2005. The TEC time series for the five quietest and five most disturbed days of each month of the year were selected for the study. The objective of the study is to have better understanding of the causes observed during day-to-day variability of ionospheric parameters so that it can be effectively used for modeling and prediction purpose.
\end{abstract}

Keywords: Global Positioning System, Total Electron Content, Quiet Days and Disturbed days, Low Latitude

\section{INTRODUCTION}

The study of the day-to-day variability of ionospheric parameters is of scientific interest in view of the causative mechanisms and of great importance in assessing prediction capabilities. The variability in TEC, which is of practical importance in communication systems, navigational control applications etc. is best described by the ratio of the standard deviation of daily TEC values from the monthly mean to the monthly mean values. Many authors have reported large day-to-day variability in daily values of TEC and tried to correlate this with magnetic activity, solar flux, or the effect of neutral winds [1]. [2] studied day to day variability using TEC of number of stations extending from equatorial to midlatitudes in Indian and American sectors for high and low solar activity period. It is found that variability is higher in American sector as compared to that in Indian and Pacific sectors. The solar activity is found to have significant control on the day-to-day variability whereas magnetic activity effect is not systematic. Under the geomagnetic disturbances variability is suppressed in nighttime and is enhanced in daytime in Indian and Pacific sectors whereas in American sector the trend is reversed.

${ }^{1}$ Department of Education in Science and Mathematics Regional Institute of Education, Bhopal, India

${ }^{2}$ Department of Physics IEHE, Bhopal, India 
[3] provides a method to investigate the monthly TEC variations in the ionospheric equatorial anomaly region by using GPS. Computing TEC from GPS data is feasible due to the dispersive nature of the ionosphere, which affects the speed of propagation of the electromagnetic waves transmitted by the GPS satellites on two $\mathrm{L}-$ band frequencies $(\mathrm{L} 1=1575.42 \mathrm{MHz}$ and $\mathrm{L} 2=1227.60 \mathrm{MHz}$ ) as they travel through that region of the atmosphere. The change in satellite-to-receiver signal propagation time due to the ionosphere is directly proportional to the integrated free electron density along the signal path. GPS pseudo range measurements are increased (the signal is delayed) and the carrier phase measurements are reduced (the phase is advanced) by the presence of the ionosphere. After forming the linear combination of these measurements on the L1 and L2 frequencies, the carrier phase and the pseudo range TEC are obtained. Both dual-frequency phase and code pseudo range observations have been used to derive the ionospheric TEC [4]. The large day-to-day variability in TEC has been observed worldwide, irrespective of geographic location, local time, and season of the year, solar flux and magnetic activity conditions. In this work, we use GPS data from Bhopal station to study the variability of the Total Electron Content during quiet and disturbed days which we use to define quantitative descriptions of variability versus local time, monthly, solar and magnetic activity for the period March 2004 - February 2005 during low solar activity period.

\section{DATA \& METHOD OF ANALYSIS}

For the present study the ionospheric data used is received from Global Positioning System (GPS) - GSV 4004A stationed at Space Science Laboratory Barkatullah University, Bhopal $\left(23.2^{\circ} \mathrm{N}, 77.6^{\circ} \mathrm{E}\right)$. In the present work hourly values of TEC were used to study the day-to-day variability of TEC during low solar activity period March 2004 - February 2005. The STEC can be converted to vertical TEC as follows:

$$
T E C=\text { slant } T E C \times \cos \chi_{m}
$$

where $\chi_{m}$ is the angle of incidence at $400 \mathrm{~km}$ altitude of a GPS ray from satellite to ground receiver. $\cos \chi$ $\mathrm{m}$, an obliquity factor, is defined as :

$$
\cos \chi_{m}=\sqrt{1-\left(\frac{h_{s p} \cos \varepsilon}{h_{s p}+R_{E}}\right)^{2}}
$$

where $\mathrm{R}_{\mathrm{E}}$ is the radius of the Earth, $\varepsilon$ is the elevation angle, $h_{s p}$ is the height of the ionospheric point, which is usually assumed to be $400 \mathrm{~km}$.

All the TEC values used for the present study have been corrected from biases using satellite and receiver bias values obtained from the data center of Space Science Laboratory, Department of Physics, Barkatullah University, Bhopal. The methods by which the instrument biases can be removed from the measured TEC values have been explained in detail in [5] [6].

The hourly data of the total electron content has been analyzed statistically and morphologically to investigate the day-to-day variability in TEC using GPS measurements occurred for the period of March 2004 - February 2005 at Bhopal during low solar activity. The events were looked at in terms of their monthly variation and their dependence upon local time, solar and magnetic activity along with geomagnetic activity control over day-to-day variability in TEC. All the characteristics of day-to-day variability in TEC were analyzed in local time.

TEC is a parameter, which is used to monitor the temporal and spatial behaviour of the ionosphere. It is subjected to significant dynamical changes, particularly in the equatorial and low 
latitude regions, owing to changes in electric fields [7]. We have considered that the standard deviation $(\sigma)$ of hourly TEC values from monthly mean value $(\mathrm{X})$ is determined, from which the ratio $(\sigma / \mathrm{X})$ in percentage is derived for each hour of each month of observation. Variability of TEC during quiet days and disturbed days is also compared. Solar flux $(10.7 \mathrm{~cm}), \mathrm{Kp}$ and Ap indices collected from the World Data Center Kyoto, Japan. Data from all satellites with elevation angle higher than $15^{\circ}$ have been used in this analysis. Although it is well known that signals from low elevation satellites suffer larger fluctuations we include data from relatively low elevation satellites in order to analyze a more realistic scenario for a GPS user. The outcomes are presented in the subsequent sections:

\section{RESULTS}

\section{A. Monthly variation of TEC with solar geophysical factors -}

The month-to-month variation of TEC as well as for other solar geophysical parameters such as solar $(10.7 \mathrm{~cm})$ flux, $\mathrm{Kp}$ and Ap indices is depicted in Figure 1 during solar minimum period March 2004 - February 2005. It is observed that during the total period variation in TEC and solar flux are well correlated i.e. solar flux has appreciable association with the variability in TEC. Figure clearly indicates that whenever magnetic activity suddenly decreases, increase in TEC values observed while at the same time solar activity is in decline phase.

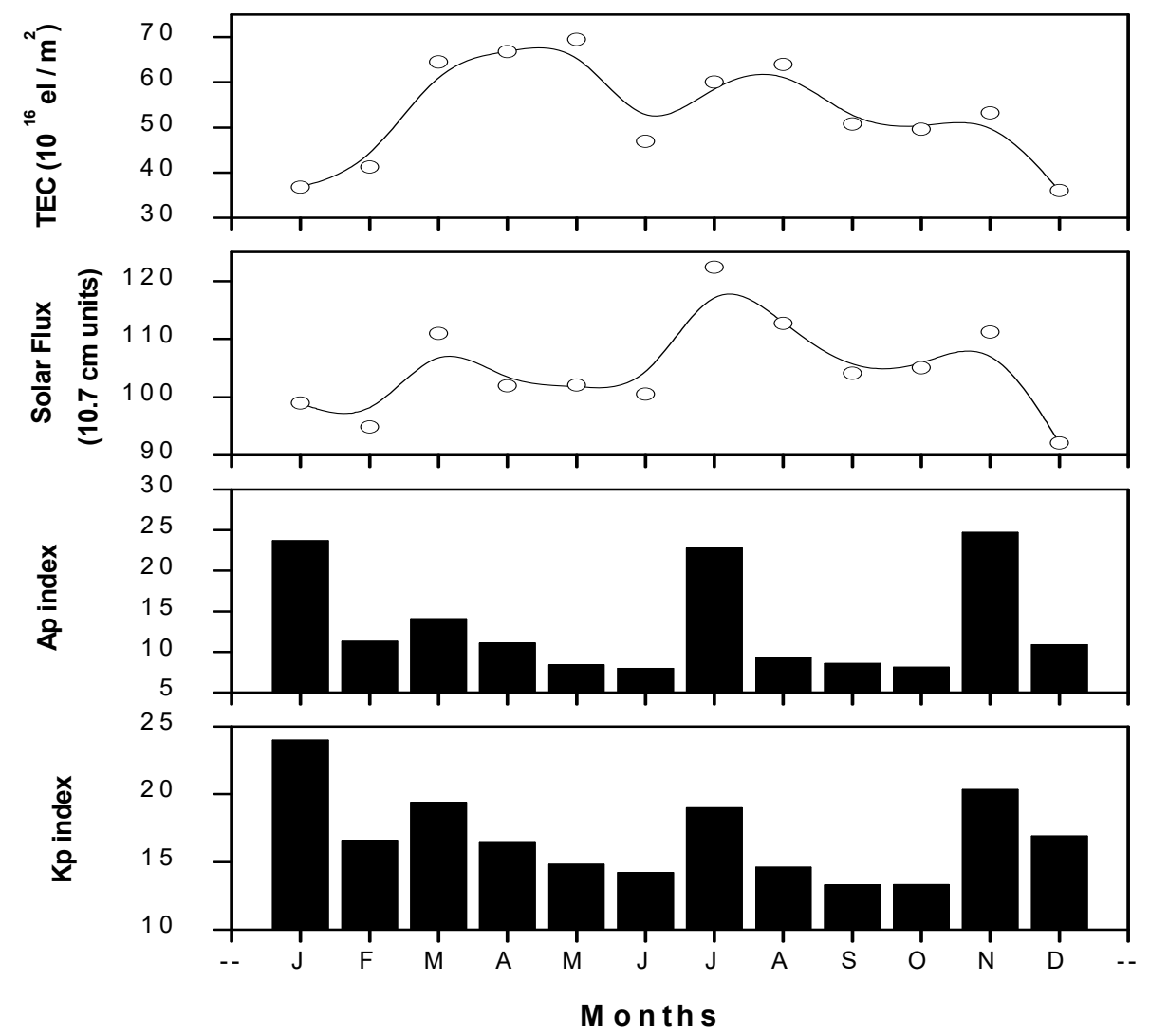

Figure 1: Monthly variation of Kp, Ap index, Solar $(10.7 \mathrm{~cm})$ Flux, and TEC for the period March 2004 - February 2005 at Bhopal (low solar activity period). 


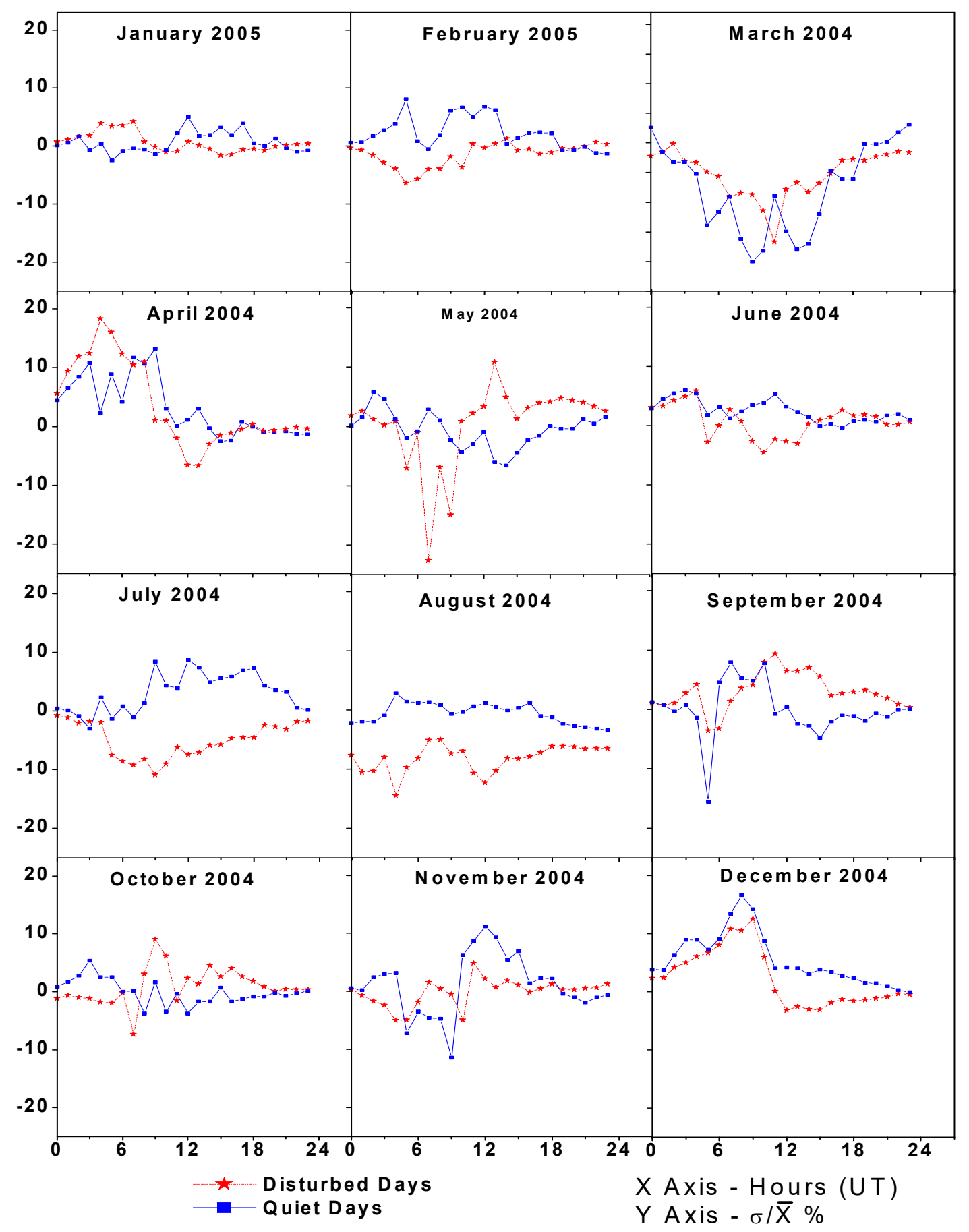

Figure 2: Average diurnal percentage variation of TEC from monthly mean for 5 QQ and DD days for each month for the period March 2004 - February 2005 at Bhopal (low solar activity period).

\section{B. Geomagnetic Activity Control over Day-to-Day Variability in TEC}

To examine the effect of magnetic disturbance two extreme cases have been considered viz., five very quiet days (QQ) and five very disturbed days (DD) of summer, winter and equinox months of the low solar activity period March 2004 - February 2005 as shown in Figure 2. The hourly TEC values 
and their percentage variation from the monthly mean values have been analyzed. It has been seen that the QQ and DD days percentage variation of TEC over Bhopal seems to be opposite in nature in some months (April, May, June, July, August and September) and in remaining it may be true at almost all the time. It seems that the varying geomagnetic conditions may influence TEC values; hence day-to-day variability in TEC observed during the study were opposite in nature during QQ and DD days. The behaviour of Q-days and D-days is influenced by the changes in plasma transport under varying geomagnetic conditions. It is observed that the variation in TEC is maximum for disturbed days and is minimum for quiet days. It is also found that the variation is maximum in nighttime hours than daytime hours.

\section{DISCUSSION}

GPS is a powerful tool to investigate ionospheric variability. TEC obtained from GPS signals are used to study ionospheric dynamics over Bhopal during low solar activity period March 2004 -February 2005. At Bhopal it is observed that TEC shows appreciable association with solar $10.7 \mathrm{~cm}$ flux. [2] observed positive correlation of TEC with the $10.7 \mathrm{~cm}$ solar flux might be due to the reduced contribution of neutral wind to loss processes during high sunspot years. The role of the field-aligned plasma flow is such that the protonosphere supplies ionization to the winter ionosphere whilst being maintained by the summer ionosphere. This may explain the lower correlation in summer months compared to winter months. The downward diffusion can be enhanced by cross L plasmaspheric compressions [8] by an electric field, which may be higher during high solar activity conditions.

Our result does not show any clear dependence on Ap at Bhopal. Solar wind affects the ionosphere by producing changes in the Earth's magnetic field and in particle precipitation. [9] using Waltair IEC data also did not find any clear dependence on Ap during low, as well as high solar activity. The absence of any significant correlation between IEC and Ap may partly be due to the averaging effect of quiet and disturbed day indices.

Our results shows that at Bhopal during quiet and disturbed days the behaviour of TEC seems to be opposite in nature (mirror image of each other). It has long been recognized that geomagnetic activity is a dominant cause of ionospheric variability. [10] studied the effect of magnetic activity on TEC, showed that variations from monthly means show a remarkably consistent anti-correlation between magnetically quiet versus disturbed days. The asymmetries they noted between the five quietest days (QQ) and the five most disturbed days (DD) are most pronounced at night. This is a purely statistical effect in the sense that small changes in F2-layer electron density result in large percentage changes when reckoned with respect to low nighttime mean values. The daytime variability, while small on a percentage basis, involves larger absolute changes in plasma content, and is therefore more significant, both physically and for applications.

The F2 region responses to the geomagnetic disturbances are rather complicated and consist of both positive and negative phases. The positive phase in most of the cases observed at equatorial latitude during magnetic disturbances. But some of the negative phases during prominent disturbances have also been observed [11]. The electric field plays a very important role in maintaining the F2 layer at equatorial region. The component of electric field that is responsible for daytime upward $\mathbf{E x B}$ drift is eastward during quiet conditions, which decreases during magnetic disturbances at equatorial region results in the reduction of ExB drift and hence increases in positive phase over equatorial region and negative phase over low latitudes [12]. Due to the peculiar magnetic field geometry at low latitude, phenomenon like Joule heating and field-aligned current are not able to produce strong vertical drift as found at high latitudes but their presence, effect the F2 region behaviour via recombination coefficient and this recombination effects depends on the electric field [13]. Thus the increase in the field leads to the depletion of electron concentration that corresponds to the negative phase. [9], studied the diurnal variation of the average percentage variation of IEC during quiet (QQ) and disturbed (DD) days. They 
found the QQ and DD (time variation of IEC) to be mirror images of each other at low latitudes for Indian stations. They reported that although no direct correlation with magnetic activity is apparent, there is a systematically higher variability on QQ than on DD days in day time hours of winter months in low solar activity period.

TEC variations observed at Bhopal appear to be controlled by the equatorial electrojet and are related to the coupling between the equatorial and low latitude ionization as a result of electrodynamic drift and diffusion. The $\mathrm{F}$ region ionization between $\pm 15^{\circ}$ dip latitude is dominantly controlled by the well known 'fountain effect'. A strong electrojet implies a larger $\mathbf{E x B}$ drift at the magnetic equator, and the development of a pronounced equatorial anomaly. Hence the observed day-to-day variability of TEC at low latitude Bhopal is a manifestation of the electrodynamic coupling between the equatorial and low latitude ionosphere. The renewed interest in ionospheric variability, as described here is a step towards improving quantitative understanding of the upper atmosphere [14].

\section{CONCLUSIONS}

In this work we discussed the day-to-day variability of the Total electron content at Bhopal during solar minimum obtained from GPS measurements over Bhopal for the period March 2004February2005. The study of day-to-day variability in TEC leads us to draw following conclusions: Nighttime variability in TEC shows its maxima in summer and minima in winter months.

1. The variability in TEC shows positive dependence on solar activity.

2. The quiet days and disturb days TEC are behaving differently, although on some occasions they seems to be the mirror image of each other at Bhopal.

3. Variation in TEC is maximum for disturbed days and minimum for quiet days.

\section{REFERENCES}

[1] Dabas, R.S., Sharma, N., Pillai, M.G.K., and Gwal, A.K., "Day-to-day variability of equatorial and low latitude F-region ionosphere in the Indian zone”, Journal of Atmospheric and SolarTerrestrial Physics, 2006 vol. 68 (11), pp. 1269-1277,.

[2] Aravindakshan, P. and Iyer, K.N., "Day to day variability in ionospheric electron content at low latitudes", Journal of Atmospheric and Terrestrial Physics, 1993, vol. 55, (11/12), pp. 1565-1573,.

[3] Tsai, H.F., Liu, J.Y., Tsai, W.H., and Liu, C.H., "Seasonal variations of the ionospheric total electron content in Asian equatorial anomaly regions", Journal of Geophysical Research, 2001, vol. 106(A12), pp. 363 - 369.

[4] Yizengaw, E., and Essex, E.A., "Use of GPS signals to study Total Electron Content of the Ionosphere during the geomagnetic storm on 22 September 1999", Proceedings of workshop on applications on radio (Wars), Australia, 2002.

[5] Klobuchar, J.A., "Ionospheric effects on GPS, in Global Positioning System: Theory and application”, vol. 1, Eds. Parkinson, B.W., and Spilker, J.J., American Institute of Aeronautics and Astronautics INC. Washington, 1996, 485-515.

[6] Breed, A.M., Goodwin, G.L., Vandenberg, A.M., Essex, E.A., Lynn, K. J.W., and Silby, J.H., "Ionospheric total electron content and slab thickness determination in Australia", Radio Science, 2000, vol. 32 (4), pp. 1635-1643.

[7] Kelly, M.C., "The Earth's ionosphere", Plasma Physics and Electrodynamics, International Geophysics series, vol. 43, Academic Press, INC, San Diego, 1989. 
[8] Mendillo, M., Papagiannis, M.D., and Klobuchar, J.A, “ Ionospheric Storms at Midlatitudes", Radio Science, 1970, vol. 5, 895.

[9] Prasad, D.S.V.V.D., and Rama Rao, P.V.S., "Day to day variability of ionospheric electron content over Waltair", Indian Journal of Radio Space Physics, 1993, vol. 22, pp. 391 - 396.

[10] Mendillo, M., and Schatten, K., "Influence of solar sector boundaries on ionospheric variability”, Journal of Geophysical Research, 1983, vol. 88, pp. 9145 - 9153.

[11] Adeniyi, J.O., "Magnetic storm effects on the morphology of the equatorial F2-layer" Journal of Atmospheric and Terrestrial Physics, 1986, vol. 48, pp. 695-702.

[12] Prolss, G.W., "Ionospheric F-region storms", in: Volland, H. (Ed.), Handbook of Atmospheric Electrodynamics, Boca Raton, FL, CRC Press, 2, 1995, vol. 2, pp. 195-248.

[13] Schunk, R.W., Raitt, W.J., Banks, P.M., "Effects of electric fields on the daytime highlatitudes E and F regions", Journal of Geophysical Research, 1975, vol. 80, 3121.

[14] Maski, K., Sarkar, S., Vijay, S. K., Jain S.K., Gwal, A.K., "GPS Based Study of Ionospheric Variability in TEC at Low Latitude Station Bhopal", International Journal of Emerging Technology and Advanced Engineering, ISSN 2250-2459, ISO 9001:2008 Certified Journal, June 2015, Vol. 5, Issue 6, pp. 104-108. 